\title{
Geometry of a naked singularity created by standing waves near a Schwarzschild horizon, and its application to the binary black hole problem
}

\author{
Ilya Mandel* \\ Theoretical Astrophysics, California Institute of Technology, Pasadena, California 91125, USA
}

(Received 30 May 2005; published 25 October 2005)

\begin{abstract}
The most promising way to compute the gravitational waves emitted by binary black holes (BBHs) in their last dozen orbits, where post-Newtonian techniques fail, is a quasistationary approximation introduced by Detweiler and being pursued by Price and others. In this approximation the outgoing gravitational waves at infinity and downgoing gravitational waves at the holes' horizons are replaced by standing waves so as to guarantee that the spacetime has a helical Killing vector field. Because the horizon generators will not, in general, be tidally locked to the holes' orbital motion, the standing waves will destroy the horizons, converting the black holes into naked singularities that resemble black holes down to near the horizon radius. This paper uses a spherically symmetric, scalar-field model problem to explore in detail the following BBH issues: (i) The destruction of a horizon by the standing waves. (ii) The accuracy with which the resulting naked singularity resembles a black hole. (iii) The conversion of the standingwave spacetime (with a destroyed horizon) into a spacetime with downgoing waves by the addition of a "radiation-reaction field." (iv) The accuracy with which the resulting downgoing waves agree with the downgoing waves of a true black-hole spacetime (with horizon). The model problem used to study these issues consists of a Schwarzschild black hole endowed with spherical standing waves of a scalar field, whose wave frequency and near-horizon energy density are chosen to match those of the standing gravitational waves of the BBH quasistationary approximation. It is found that the spacetime metric of the singular, standing-wave spacetime, and its radiation-reaction-field-constructed downgoing waves are quite close to those for a Schwarzschild black hole with downgoing waves - sufficiently close to make the BBH quasistationary approximation look promising for non-tidally-locked black holes.
\end{abstract}

PACS numbers: 04.25. $-\mathrm{g}, 04.25 . \mathrm{Nx}, 04.30 .-\mathrm{w}$

\section{INTRODUCTION AND SUMMARY}

It is very important, in gravitational astronomy, to have accurate computations of the gravitational waves from the inspiral of a black-hole binary [1]. However, computing these waves is extremely challenging: for the last $\approx 25$ cycles of inspiral waves, post-Newtonian approximations fail [2], and numerical relativity cannot yet evolve the full dynamical equations in this regime. It appears that the best hope for accurately computing the binary black hole (BBH) inspiral waves is by a quasistationary approximation $[3,4]$. In this approximation, the energy and angular momentum of the binary are conserved by the imposition of standing gravitational waves, and the spacetime has a helical Killing vector field. The standing-wave radiation required to keep the orbit stationary is computed by demanding that the energy contents of the gravitational waves (GW) be minimized [4].

Standing-wave radiation consists of a sum of ingoing and outgoing radiation at infinity, and downgoing and upgoing radiation at the black-hole horizons. The physical spacetime, with purely outgoing waves at infinity and downgoing waves at the horizons, can be recovered from the standing-wave spacetime by adding a perturbative radiation-reaction field [5]. The solution for the $\mathrm{BBH}$ inspiral consists of a series of quasistationary solutions that

*Electronic address: ilya@caltech.edu evolve from one to another via energy and angular momentum loss triggered by the radiation-reaction field. The waves measured at a detector can be deduced from this sequence of quasistationary solutions.

The black holes comprising the binary are tidally locked if their horizon generators are static in the frame corotating with the orbit. In the tidally locked case, the metric perturbations necessary to keep the black holes on a stationary orbit are static in the corotating frame, and the black holes can be regarded as having bifurcate Killing horizons (both a past horizon and a future horizon).

In reality, the black holes are not tidally locked. Their mutual tidal forces are not strong enough to maintain locking during the inspiral. In the absence of tidal locking, the standing waves of the standing-wave approximation destroy the black-hole horizons: the downgoing waves destroy the past horizon by building up an infinite energy density at the past horizon, and the upgoing waves destroy the future horizon. Therefore, we expect that forcing the orbit to be stationary via the addition of standing gravitational waves will strip the Kerr black holes of their horizons and leave naked singularities in their place [6].

Despite this radical change in the character of the orbiting bodies, it is reasonable to expect that the standing-wave solution will give a quite accurate approximation to the true physical black-hole spacetimes everywhere except very near the black-hole horizons. In order to verify or refute this expectation, it is necessary to explore the nature 
of the singularities created by the standing gravitational waves and to test how well the physical solution with true black holes can be extracted from the standing-wave solution with naked singularities.

As a first step in such an exploration, we consider in this paper a simple model problem designed to give insight into the nature of the singularities generated by the standing gravitational waves, and the accuracy with which the physical, BBH spacetime can be recovered from the standing-wave, singularity-endowed spacetime.

Our model problem is a single, spherically symmetric black hole that is converted into a naked singularity by spherical standing waves of a scalar field.

We begin our analysis in Sec. II by describing the mapping between the BBH problem, into which we seek insight, and our spherical, scalar-field model problem. In particular, we deduce what should be the range of scalarfield amplitudes and frequencies in order to mock up the gravitational waves of the $\mathrm{BBH}$ problem.

Then in Sec. III, we construct and explore the standingwave spacetime for our spherical model problem. We initially treat the standing-wave scalar field as residing in the unperturbed Schwarzschild spacetime of the black hole, and we use Regge-Wheeler first-order perturbation theory to compute the scalar-energy-induced deviations of the hole's metric from Schwarzschild. The metric perturbations consist of a static component and a component varying in time at twice the scalar-field frequency (see Fig. 2 below). The oscillatory component is smaller than the static one and higher-order harmonics of both the field and the metric are strongly suppressed.

The static metric perturbation grows divergently as one approaches the Schwarzschild horizon-an obvious indication of the horizon's destruction by the standing-wave stress energy. To explore the structure of the resulting naked singularity, in Sec. III B we abandon perturbation theory and switch to the fully nonlinear, coupled Einstein equations and scalar-field equations. To simplify the analysis, we focus solely on the static part of the singularity's metric; we do this by time averaging the scalar stressenergy tensor before inserting it into the fully nonlinear Einstein equations. We solve the resulting equations numerically to obtain the spacetime geometry outside the singularity. The geometry's embedding diagram (Fig. 3 below) and the redshift seen by a distant observer (Fig. 4 below) show that the spacetime remains nearly Schwarzschild outside the Schwarzschild horizon, but deviates strongly from Schwarzschild at $r \approx 2 M$ and below. (Here $M$ is the mass of the holelike singularity and we use geometrized units $c=G=1$ everywhere in this paper.) Above $r=2 M$, the standing-wave spacetime is very nearly identical to the Schwarzschild spacetime down to radii that are well inside the inner edge of the effective potential (Fig. 5). Below $r=2 M$, radial distance changes far more slowly than areal radius; i.e., $g_{r r}$ tends to 0 as $r \rightarrow$
0 . The redshift seen by an external observer rises rapidly when the emitter falls inside $r=2 M$. However, a signal from the singularity at $r=0$ may be infinitely redshifted or infinitely blueshifted, depending on the choice of scalarfield parameters.

In Sec. IV we turn to the model spherical spacetime that mocks up our desired BBH solution: the spacetime of a Schwarzschild black hole with downgoing scalar waves. Not surprisingly, the metric perturbations induced by the downgoing scalar-wave energy are those of the Vaidya solution of Einstein's equations - a slowly growing black hole with a smooth, nonsingular future horizon. This spacetime is well approximated, for short time intervals, by the Schwarzschild solution with (constant) Schwarzschild mass equal to the instantaneous Vaidya mass.

Finally, in Sec. V we demonstrate that by adding a perturbative radiation-reaction field to the standing-wave solution, a downgoing solution to the scalar-wave equation can be recovered. We explore the level of agreement between these downgoing waves that live in the singularity-endowed standing-wave spacetime and the downgoing waves in the Schwarzschild approximation to the Vaidya spacetime. The agreement (for details see Sec. V and Fig. 6 below) is rather good for scalar-wave amplitudes and frequencies that mock up the BBH problem-sufficiently good to give optimism that the standingwave approximation will give accurate gravitational waveforms for the final stages of binary-black-hole inspiral.

\section{THE MAPPING BETWEEN THE BBH PROBLEM AND OUR MODEL SCALAR-FIELD PROBLEM}

In our exploration of the quasistationary, standing-wave approximation for black-hole binaries we shall study several spherically symmetric spacetimes, each endowed with a standing-wave scalar field. In Sec. III A the spacetime will be Schwarzschild, or Schwarzschild with first-order gravitational perturbations generated by the scalar-field stress-energy tensor. In Sec. III B the spacetime will be that of a naked singularity generated by the coupled, timeaveraged Einstein-scalar-field equations. In this section we shall identify the parameter regime relevant to gaining insight from these spacetimes into the binary-black-hole problem.

In each of these spherical spacetimes, the scalar field must be a solution to the wave equation:

$$
\square \Phi=\frac{1}{\sqrt{-g}}\left(\sqrt{-g} g^{\alpha \beta} \Phi_{, \alpha}\right)_{, \beta}=0,
$$

where $g_{\alpha \beta}$ is the spacetime metric with the interval

$$
d s^{2}=f(r, t) d t^{2}+g(r, t) d r^{2}+r^{2}\left(d \theta^{2}+\sin ^{2} \theta d \phi^{2}\right) .
$$

We assume that the scalar field is monochromatic with frequency $\omega$, and we write it in the form 


$$
\Phi=\mathfrak{R}\left(\frac{\Psi(r) e^{-i \omega t}}{r}\right),
$$

where $\Re()$ denotes the real part and the phase was set by the choice of the zero of time $t$.

The scalar field $\Phi$ serves as the source of curvature in the Einstein equations,

$$
G_{\alpha \beta}=8 \pi T_{\alpha \beta},
$$

where the stress-energy tensor depends on the scalar field according to

$$
T_{\alpha \beta}=\frac{1}{4 \pi} \Phi_{, \alpha} \Phi_{, \beta}-\frac{1}{8 \pi} g_{\alpha \beta} \Phi_{, \mu} \Phi^{, \mu}
$$

[cf. Eq. (20.66) of [7] or Eq. (A.11) of [8]].

We can rewrite Eqs. (4) and (5) in a simpler form via the Ricci tensor:

$$
R_{\alpha \beta}=2 \Phi_{, \alpha} \Phi_{, \beta} .
$$

Relevant ranges for the scalar-field frequency and amplitude are determined by the binary-black-hole problem we are modeling. Suppose that the black holes in the binary have equal mass $M$, and let $a$ be their radial separation. Since we are interested in the late inspiral, where the postNewtonian methods fail, the desired range of parameters should correspond to $6 \lesssim a / M \lesssim 15$ [2].

The Keplerian orbital frequency of the black holes is

$$
\Omega=\frac{1}{M} \sqrt{\frac{2}{(a / M)^{3}}} .
$$

The gravitational wave frequency is twice the Keplerian frequency, and we set our scalar-field frequency equal to the GW frequency:

$$
\omega=2 \Omega=\frac{2}{M} \sqrt{\frac{2}{(a / M)^{3}}} .
$$

The power going down a black hole due to the orbital motion of its companion is approximately

$$
P_{\mathrm{GW}}=\frac{32}{5} M^{4} \mu^{2} \Omega^{6},
$$

where $\mu$ is the mass of the companion $[9,10]$. Although the calculations in Refs. $[9,10]$ underlying Eq. (9) were carried out under the assumption $\mu \ll M$, we will use Eq. (9) to approximate the power for equal mass black holes, $\mu=$ $M$. This approximation is not too worrisome because we are interested in the general features of the scalar-field model, which roughly corresponds to the interesting range of BBH separations, rather than in the quantitative results for this model. We select the scalar-field amplitude by demanding that its energy density near the horizon equal the GW energy density there:

$$
\frac{d E}{d V} \approx \frac{P_{\mathrm{GW}}}{4 \pi(2 M)^{2}} .
$$

(In the spirit of this approximate analysis we here ignore the gravitational blueshift of the energy.) By equating this energy density to the value of $T_{00}$ at the horizon, computed by inserting Eq. (3) into Eq. (5), we obtain the scalar-field amplitude inside the peak of the effective potential:

$$
\Psi_{\text {in }}=\sqrt{\frac{64}{5}}\left[\frac{1}{(a / M)}\right]^{3} M .
$$

Using Eqs. (8) and (11), we can compute the desired scalar-field frequency and amplitude for the boundaries of the region of interest:

$$
\begin{aligned}
& a=6 M \Rightarrow \omega \approx 0.19 / M, \quad \Psi_{\mathrm{in}} \approx 0.017 M ; \\
& a=15 M \Rightarrow \omega \approx 0.049 / M, \quad \Psi_{\mathrm{in}} \approx 0.0011 M .
\end{aligned}
$$

\section{STANDING-WAVE SCALAR FIELD}

We now turn to the standing-wave scalar-field spacetime that mocks up the spacetimes of the BBH standing-wave approximation. The metric of this spacetime has the form of Eq. (2) and the standing-wave scalar field follows from Eq. (3):

$$
\Phi=\frac{\Psi(r) \cos \omega t}{r},
$$

where $\Psi(r)$ is now real.

We shall treat the standing-wave scalar field twice via two different simplifying assumptions. First, in Sec. III A, we will consider the scalar field perturbatively; its wave equation will be that of the Schwarzschild spacetime, and its stress energy will generate first-order perturbations of the metric away from Schwarzschild. Then in Sec. III B, we will consider the fully nonlinear Einstein-scalar-field spacetime but with the scalar stress energy averaged over time to make the metric static.

\section{A. Perturbative standing-wave solution \\ 1. Perturbative formalism for the standing-wave spacetime}

In our first approach, the lowest-order solution for the scalar field is computed by solving the wave equation (1) in the Schwarzschild background with the metric

$$
\begin{aligned}
d s^{2} & =g_{\alpha \beta}^{B} d x^{\alpha} d x^{\beta} \\
& =-(1-2 / r) d t^{2}+\frac{1}{1-2 / r} d r^{2}+r^{2} d \Omega^{2},
\end{aligned}
$$

where we rescale so that $M=1$. The wave equation simplifies as follows [cf. Eq. (32.27b) of [7]]:

$$
\frac{d^{2} \Psi}{d r^{* 2}}=\left[-\omega^{2}+(1-2 / r) \frac{2}{r^{3}}\right] \Psi,
$$

where $r^{*}$ is the Regge-Wheeler tortoise coordinate [11],

$$
r^{*}=r+2 \ln (r / 2-1) .
$$


Because $\omega^{2}$ dominates the right-hand side of Eq. (15) both far from the horizon $(r \gg 2)$ and very near the horizon, the scalar field will oscillate with a nearly constant frequency $\omega$ in those regions. In between, where the effective potential

$$
V\left(r^{*}\right)=(1-2 / r)\left(2 / r^{3}\right)
$$

is significant, there is an intermediate transitional region (see Fig. 1). (In this paper we mention several times "the inner edge of the peak of the effective potential"; we define this inner edge to be the radius at which the effective potential drops to $1 \%$ of its maximum value at the peak.)

Since we are approaching the problem perturbatively, we are interested in some small metric perturbation $h_{\alpha \beta}$ on top of the background metric $g_{\alpha \beta}^{B}$ of Eq. (14) that would yield the curvature corresponding to the stress-energy tensor of the scalar field:

$$
g_{\alpha \beta}=g_{\alpha \beta}^{B}+h_{\alpha \beta}
$$

Linearizing in $h_{\alpha \beta}$, this metric gives the Ricci tensor

$$
R_{\alpha \beta}=R_{\alpha \beta}^{B}+\frac{1}{2}\left(h_{\mu \alpha \mid \beta}{ }^{\mu}+h_{\mu \beta \mid \alpha}{ }^{\mu}-h_{\alpha \beta \mid \mu}{ }^{\mu}-h_{\mid \alpha \beta}\right),
$$

where $h=h_{\mu}{ }^{\mu}$ and | represents the covariant derivative in the background metric $g_{\alpha \beta}^{B}$. For the Schwarzschild background metric, $R_{\alpha \beta}^{B}=0$.

We are interested only in spherically symmetric perturbations. A gauge transformation brings additional simplification, so $h_{\alpha \beta}$ can be written in the following simple Regge-Wheeler form:

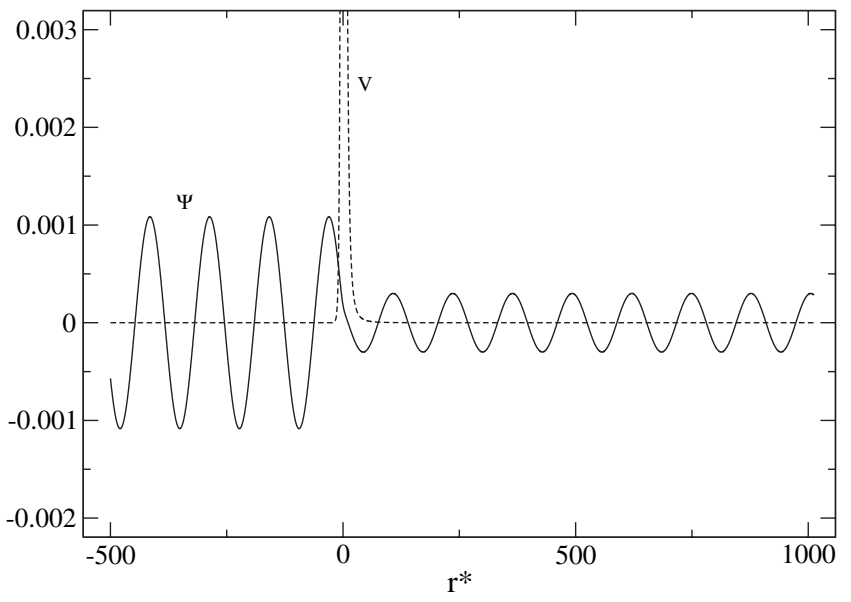

FIG. 1. The standing-wave scalar field in a Schwarzschild background (solid curve) and the effective potential (dashed curve) for angular frequency $\omega=0.049$.

$$
h_{\alpha \beta}=\left(\begin{array}{cccc}
(1-2 / r) H_{0}(t, r) & 0 & 0 & 0 \\
0 & \frac{H_{2}(t, r)}{1-2 / r} & 0 & 0 \\
0 & 0 & 0 & 0 \\
0 & 0 & 0 & 0
\end{array}\right) .
$$

[Compare with Eq. (13) of [11] for the case $L=0$.]

We can now substitute $h_{\alpha \beta}$ given by Eq. (20) into Eq. (19) to compute the perturbed Ricci tensor:

$$
\begin{aligned}
R_{t t}= & -\frac{1}{2} \frac{\partial^{2}}{\partial t^{2}} H_{2}-\frac{r-2}{2 r^{3}}\left[(2 r-1) \frac{\partial}{\partial r} H_{0}\right. \\
& \left.+\frac{\partial}{\partial r} H_{2}+r(r-2) \frac{\partial^{2}}{\partial r^{2}} H_{0}\right] ; \\
R_{t r}= & \frac{1}{r} \frac{\partial}{\partial t} H_{2} ; \\
R_{r r}= & \frac{r^{2}}{2(r-2)^{2}} \frac{\partial^{2}}{\partial t^{2}} H_{2}+\frac{1}{2 r(r-2)}\left[3 \frac{\partial}{\partial r} H_{0}\right. \\
& \left.+(2 r-3) \frac{\partial}{\partial r} H_{2}+r(r-2) \frac{\partial^{2}}{\partial r^{2}} H_{0}\right] ; \\
R_{\theta \theta}= & H_{2}+\frac{r-2}{2} \frac{\partial}{\partial r} H_{0}+\frac{r-2}{2} \frac{\partial}{\partial r} H_{2} .
\end{aligned}
$$

Inserting expressions (21) for $R_{\alpha \beta}$ into the Einstein equations (6), one obtains a set of rather complicated partial differential equations (PDE's) containing both spatial and time derivatives to the second order. However, we expect that the equations can be further simplified because of additional consistency conditions imposed on $\Phi$ by the wave equation (15). Indeed, after adding the $R_{t t}$ and $R_{r r}$ equations with appropriate coefficients to remove the second derivatives in both $t$ and $r$, and using $R_{\theta \theta}=0$ to relate $H_{0}$ to $H_{2}$, we obtain the following set of first-order ordinary differential equations for $\mathrm{H}_{0}$ and $\mathrm{H}_{2}$ :

$$
\begin{aligned}
\frac{\partial H_{2}}{\partial r} & =-\frac{H_{2}}{r-2}+\frac{r^{3}}{(r-2)^{2}} \Phi_{, t} \Phi_{, t}+r \Phi_{, r} \Phi_{, r} ; \\
\frac{\partial H_{0}}{\partial r} & =-\frac{\partial H_{2}}{\partial r}-\frac{2}{r-2} H_{2} .
\end{aligned}
$$

These far simpler equations can be shown to produce no spurious solutions; in fact, together with the wave equation (15), they are equivalent to the second-order PDE system (6) and (21).

\section{First-order metric perturbations due to the standing-wave scalar field}

In the scalar-field ansatz (13) we assumed $\Phi \propto \cos \omega t$. Therefore, the driving term on the right-hand side of Eq. (22a) will have static components as well as components oscillating in time at the frequency $2 \omega$. Because there is no mixing of terms with distinct time signatures in Eqs. (22), these terms may be treated separately:

$$
\begin{aligned}
& H_{2}(t, r)=H_{2}^{\text {stat }}(r)+H_{2}^{\cos }(r) \cos 2 \omega t ; \\
& H_{0}(t, r)=H_{0}^{\text {stat }}(r)+H_{0}^{\cos }(r) \cos 2 \omega t .
\end{aligned}
$$


(There is no $\sin 2 \omega t$ term with our particular choice of the scalar-field phase.)

For $r \gg 2$ analytical approximations for $H_{0}$ and $H_{2}$ are easy to obtain because the scalar field is particularly simple there:

$$
\Phi \approx\left(\Psi_{0} / r\right) \cos \left(\omega r^{*}\right) \cos (\omega t),
$$

where $\Psi_{0}$ is the scalar-field amplitude as $r \rightarrow \infty$. Inserting this into Eqs. (22), we readily compute, at large $r$ :

$$
\begin{aligned}
& H_{2}^{\text {stat }}(r) \approx \frac{1}{2} \omega^{2} \Psi_{0}^{2}-\frac{\Psi_{0}^{2}}{4 r^{2}}-\frac{\Psi_{0}^{2} \cos 2 \omega r^{*}}{4 r^{2}} ; \\
& H_{2}^{\cos }(r) \approx-\frac{\Psi_{0}^{2}}{4 r^{2}}-\frac{\Psi_{0}^{2} \cos 2 \omega r^{*}}{4 r^{2}}-\frac{\Psi_{0}^{2} \omega \sin 2 \omega r^{*}}{4 r} ; \\
& H_{0}^{\text {stat }}(r) \approx-\omega^{2} \Psi_{0}^{2} \ln r+\frac{\Psi_{0}^{2} \cos 2 \omega r^{*}}{4 r^{2}} ; \\
& H_{0}^{\cos }(r) \approx \frac{\Psi_{0}^{2} \cos 2 \omega r^{*}}{4 r^{2}}+\frac{\Psi_{0}^{2} \omega \sin 2 \omega r^{*}}{4 r}
\end{aligned}
$$

The static components of $H_{2}$ and $H_{0}$ are nonvanishing at infinity, and $H_{0}^{\text {stat }}$ actually diverges. This indicates that, due to the energy contained in the scalar field, the spacetime is not asymptotically flat. However, this bad behavior at infinity is an artifact of our model problem and is irrelevant to the issues we are studying in this paper.

A more significant issue for the binary-black-hole problem is the contribution of the additional energy stored in standing gravitational waves inside the orbit of a companion to the effective mass seen by the companion and the resulting change in the companion's angular velocity. Translating this issue into the language of our model problem, we ask for the energy stored in standing waves of the scalar field inside the radius $a$ and the value of the metric perturbation $H_{0}$ there. For scalar-field amplitude and frequency corresponding to the inner boundary of the region of interest $a=6 M$ [Eq. (12a)], the energy stored in the standing waves of the scalar field between $r=2.01 \mathrm{M}$ and $r=6 M$ (obtained by integrating $R_{00}$ ) is $E \approx 10^{-4} M$ and $H_{0}(r=6 M) \approx 10^{-5}$ [cf. Eqs. (24)]. For scalar-field parameters corresponding to the outer boundary of the region of interest $a=15 M$ [Eq. (12b)], the energy stored in the scalar waves between $r=2.01 M$ and $r=15 M$ is $E \approx 10^{-8} M$ and $H_{0}(r=15 M) \approx 10^{-8}$. This suggests that the presence of standing waves should not significantly affect the determination of the angular velocity of the binary or the gravitational wave frequency.

We can read off from Eqs. (24) the ratios of the oscillatory and static components of the metric perturbations at large $r$. They are

$$
\left|\frac{H_{2}^{\text {cos }}}{H_{2}^{\text {stat }}}\right| \approx \frac{1}{2 \omega r}
$$

and

$$
\left|\frac{H_{0}^{\cos }}{H_{0}^{\text {stat }}}\right| \approx \frac{1}{4 \omega r \ln r}
$$

thus, the static components dominate far from the horizon.

Equations (24) can be used to set initial conditions for the metric perturbations at some large $r$, allowing for a numerical solution to Eqs. (22) from there down to the horizon, $r=2$. The resulting solution, plotted in Fig. 2, indicates that static components continue to dominate near the horizon.

Near the horizon (inside the effective-potential peak), the scalar field has the form

$$
\Phi \approx\left(\Psi_{\text {in }} / 2\right) \cos \omega r^{*} \cos \omega t,
$$

where $\Psi_{\text {in }}$ is the scalar-field amplitude as $r \rightarrow 2$. Inserting this approximation into Eq. (22a) and averaging the righthand side leads to the following rough estimate of the magnitude of the perturbation near the horizon:

$$
H_{2}^{\text {stat }} \approx \frac{2 \omega^{2} \Psi_{\mathrm{in}}^{2} \ln (r-2)}{r-2} .
$$

Inverting this formula can give a useful estimate of the distance from the horizon where the perturbation reaches a particular value; the estimate turns out to be accurate to within a factor of 2 .

Although it appears that the metric perturbation diverges at the expected location of the horizon, our perturbative solution is not trustworthy in this regime for several reasons in addition to the obvious one of violating the perturbative assumption $H_{0}, H_{2} \ll 1$ :

(1) We ignored the backreaction, i.e., the feedback of the metric perturbation into the wave equation. Using the Schwarzschild metric in place of the more accurate perturbed metric in the wave equation, that is, using the approximate Eq. (15) in place

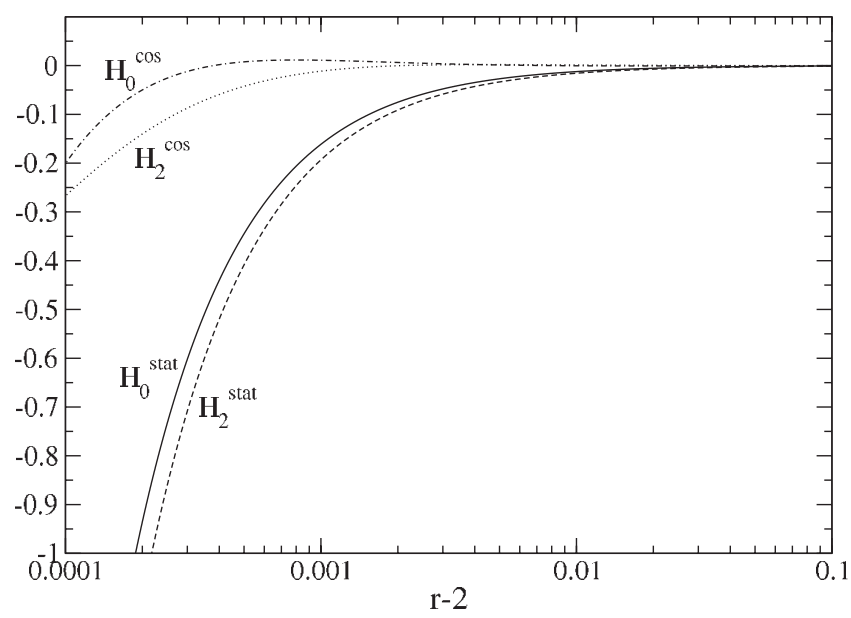

FIG. 2. Metric perturbations for a standing-wave scalar field in a Schwarzschild background with angular frequency $\omega=0.19$ and amplitude $\Psi_{0}=0.015$ far from the black hole, corresponding to a binary separation $a \approx 6 M$ [Eq. (12a)]. 
of the exact Eq. (1), is equivalent to an error in the scalar-field frequency $\Delta \omega / \omega \approx O(H)$, which produces phase offsets in the scalar field when the wave equation is integrated numerically.

(2) We linearized the Ricci tensor in the perturbations, neglecting higher-order $O\left(H^{2}\right)$ effects. In contrast to the linearized equations (22) for $H_{2}$ and $H_{0}$, the nonlinear perturbative equations are

$$
\begin{aligned}
\frac{\partial H_{2}}{\partial r}= & -\frac{H_{2}\left(1+H_{2}\right)}{r-2}+\frac{r^{3}}{(r-2)^{2}} \frac{\left(1+H_{2}\right)^{2}}{1-H_{0}} \\
& \times\left(\Phi_{, t}\right)^{2}+r\left(1+H_{2}\right)\left(\Phi_{, r}\right)^{2} ; \\
\frac{\partial H_{0}}{\partial r}= & \left(1-H_{0}\right)\left[-\frac{1}{1+H_{2}} \frac{\partial H_{2}}{\partial r}-\frac{2 H_{2}}{r-2}\right] .
\end{aligned}
$$

Linearization introduces local errors of order $H$ into the Einstein equations. However, the errors can build up globally when the equations are integrated to obtain a numerical solution. The errors produced by linearizing the Ricci tensor (the differences between solutions to the linearized and nonlinear Einstein equations without backreaction in the wave equation) have the same order of magnitude in the parameter range of interest as the errors produced by neglecting backreaction [the differences between solutions to the nonlinear Einstein equations depending on whether wave equation (15) or (1) is used].

(3) We ignored higher harmonics of the scalar field and of the metric perturbations that would arise from the backreaction. However, these higher harmonics are suppressed by additional factors of $H \propto \Psi^{2}$ : whereas the static and $\cos 2 \omega t$ components of $H$ are quadratic in $\Psi$, higher-order harmonics of frequency $2 n \omega$ are proportional to $\Psi^{2 n}$ for $n>1$.

\section{B. Time-averaged fully nonlinear standing-wave solution}

To explore the behavior of the standing-wave spherical scalar field and the spherical metric near and inside the expected location of the horizon, we solve the fully nonlinear coupled Einstein-scalar-field equations including full backreaction in the wave equation. To simplify our solution, we average the stress-energy tensor in time to remove oscillations of the scalar-field energy, so that the metric is static. This is justified by the perturbative analysis above, which demonstrates that metric components oscillating in time are smaller than static metric components and largely decouple from them.

\section{Formalism for nonlinear solution with backreaction}

We write the static spherically symmetric metric in the form

$$
d s^{2}=-e^{\beta(r)+\alpha(r)} d t^{2}+e^{\beta(r)-\alpha(r)} d r^{2}+r^{2} d \Omega^{2},
$$

and we compute the Einstein tensor from this metric in the standard way. The Einstein tensor is diagonal and its angular components $G_{\hat{\theta} \hat{\theta}}$ and $G_{\hat{\phi} \hat{\phi}}$ are not particularly interesting because of spherical symmetry (the angular components of the Einstein equations will merely repeat the time and radial components by virtue of the contracted Bianchi identities). The careted subscripts $\hat{\mu}$ denote the orthonormal basis associated with the $(t, r, \theta, \phi)$ coordinate system. The relevant nonvanishing terms of the Einstein tensor in the orthonormal basis are

$$
\begin{aligned}
G_{\hat{t} \hat{t}} & =e^{\alpha-\beta}\left(\beta^{\prime}-\alpha^{\prime}\right) / r+\left(1-e^{\alpha-\beta}\right) / r^{2}, \\
G_{\hat{r} \hat{r}} & =e^{\alpha-\beta}\left(\beta^{\prime}+\alpha^{\prime}\right) / r-\left(1-e^{\alpha-\beta}\right) / r^{2},
\end{aligned}
$$

where ' denotes a derivative with respect to $r$, not $r^{*}$.

Substituting the Einstein tensor (29) and the stressenergy tensor (5) into the Einstein equations (4), we obtain:

$$
\begin{aligned}
\alpha^{\prime} & =\frac{1}{r}\left(e^{\beta-\alpha}-1\right), \\
\beta^{\prime} & =r e^{-2 \alpha}\left(\Phi_{, t}\right)^{2}+r\left(\Phi_{, r}\right)^{2} .
\end{aligned}
$$

We can now insert the standing-wave scalar-field ansatz (13) and time average the right-hand side of Eq. (30b) over a complete period. For numerical analysis it will be useful to switch to a logarithmic coordinate that changes more gradually than $r$ in the vicinity of the Schwarzschild horizon. The following generalization of the Regge-Wheeler tortoise coordinate $r^{*}$ proves convenient:

$$
\frac{d r}{d r^{*}}=e^{\alpha} .
$$

In terms of this coordinate, the wave equation (1) simplifies to

$$
\frac{d^{2} \Psi}{d r^{* 2}}=-\omega^{2} \Psi+\frac{e^{\alpha}}{r} \frac{d \alpha}{d r^{*}} \Psi
$$

and the Einstein equations (30) with time-averaged $\left(\Phi_{, t}\right)^{2}$ and $\left(\Phi_{, r}\right)^{2}$ become

$$
\begin{gathered}
\frac{d \alpha}{d r^{*}}=\frac{e^{\beta}-e^{\alpha}}{r}, \\
\frac{d \beta}{d r^{*}}=\frac{e^{-\alpha}}{2 r}\left[\Psi^{2} \omega^{2}+\left(\frac{d \Psi}{d r^{*}}\right)^{2}\right]+\frac{\Psi^{2} e^{\alpha}}{2 r^{3}}-\frac{\Psi}{r^{2}} \frac{d \Psi}{d r^{*}} .
\end{gathered}
$$

\section{Singular standing-wave spacetime}

We have solved the coupled equations (31) numerically to high accuracy for values of the scalar-field amplitude and frequency in the range relevant to the BBH problem [Eqs. (12)]. Our numerical solutions are very well approximated by analytic formulas that rely on dividing space $0<$ $r<\infty$ into three regions. Region I is "perturbed 
Schwarzschild," i.e., the region where the perturbative solution is valid $(r>2, H \lesssim 0.1)$. Region III describes the space very close to $r=0$ where the $1 / r$ terms diverge. Finally, the intermediate region II extends from the inner boundary of region I to the outer boundary of region III.

For sufficiently small amplitudes of the scalar field, the contributions from the backreaction (by which we mean the impact of the deviation of the spacetime from Schwarzschild on the solution to the wave equation) and from nonlinearity remain small until very close to $r=2$, so that the metric can be well approximated by perturbations on top of the Schwarzschild metric. In other words, the perturbative solution developed in Sec. III A is valid throughout region I. Indeed, for scalar-field amplitudes and frequencies in the range of interest, the metric perturbations $H_{0}^{\text {stat }}$ and $H_{2}^{\text {stat }}$ derived in the previous subsection match the values of $\mathrm{H}_{0}$ and $\mathrm{H}_{2}$ corresponding to the complete nonlinear solution with backreaction to within $3 \%$ for $H \lesssim 0.01$.

We begin the analysis in region III, where $r \ll 1$, by assuming $e^{\beta-\alpha} \ll 1$ as $r \rightarrow 0$, which corresponds to $g_{r r} \rightarrow$ 0 at $r=0$. (This assumption, which can be deduced from the behavior of $d \beta / d r^{*}$ in the transition region, will be shown to be self-consistent; more importantly, it is supported by our numerical solutions.) Then, from Eq. (31c), $\alpha^{\prime} \equiv d \alpha / d r \rightarrow-1 / r$, so $\alpha$ is given by

$$
\alpha=-\ln r+\alpha_{0} .
$$

Here $\alpha_{0}$ is a constant whose value depends on the amplitude and the frequency of the scalar waves; it can be roughly approximated by

$$
\alpha_{0} \sim \ln \left(\Psi_{\text {in }}^{2} \omega^{2}\right) .
$$

The wave equation (31b) becomes

$$
\begin{aligned}
\Psi^{\prime \prime} & =-\Psi \omega^{2} e^{-2 \alpha}-\alpha^{\prime}\left(\Psi^{\prime}-\Psi / r\right) \\
& =-\Psi \omega^{2} e^{-2 \alpha_{0}} r^{2}+1 / r\left(\Psi^{\prime}-\Psi / r\right) .
\end{aligned}
$$

Since we are interested in the region $r \rightarrow 0$, the last term dominates, so that the approximate solution to Eq. (32c) is

$$
\Psi=n r+k r \ln r,
$$

where $n, k$ are constants.

Substituting $\Psi$ and $\alpha$ into Eq. (31d) and selecting nonvanishing terms with the highest order in $1 / r$, we find that $\beta^{\prime} \rightarrow k^{2} /(2 r)$, so

$$
\beta=\frac{k^{2}}{2} \ln r+\beta_{0},
$$

where $\beta_{0}$ is a constant. Thus, we see that our assumption, $e^{\beta-\alpha} \ll 1$ as $r \rightarrow 0$, is self-consistent:

$$
\beta-\alpha=\left(\frac{k^{2}}{2}+1\right) \ln r+\beta_{0}-\alpha_{0} \rightarrow-\infty \text { as } r \rightarrow 0,
$$

since the coefficient of $\ln r$ is always positive.
Our numerical solution in region III agrees well with the asymptotic analytical solution (32). For instance, the value of $k$ obtained from matching $\Psi$ to the form of Eq. (32d) agrees with the value of $k$ obtained from matching $\beta$ to Eq. (32e) to one part in ten thousand. Of particular interest are the metric components and the Ricci scalar, whose asymptotics for $r \rightarrow 0$ are

$$
\begin{gathered}
g_{t t}=-e^{\beta+\alpha}=-e^{\beta_{0}+\alpha_{0}} r^{k^{2} / 2-1}, \\
g_{r r}=e^{\beta-\alpha}=e^{\beta_{0}-\alpha_{0}} r^{k^{2} / 2+1},
\end{gathered}
$$

and

$$
R=R_{\gamma}^{\gamma}=2 \Phi_{, \gamma} \Phi^{, \gamma}=k^{2} e^{\alpha_{0}-\beta_{0}} r^{-3-k^{2} / 2} .
$$

The exponent of $r$ in Eq. (32i) is always negative, so the Ricci curvature scalar tends to infinity as $r \rightarrow 0$, i.e., the radius of curvature vanishes at the singularity at $r=0$, as expected. The exponent of $r$ in Eq. (32h) is always positive, so $g_{r r}$ tends to zero as $r \rightarrow 0$ according to a power law. However, the sign of the exponent of $r$ in Eq. (32g) depends on the value of $k$, which in turn is a complicated function of the scalar-field frequency and amplitude. For some scalar-field parameter values in the range relevant to the BBH problem [Sec. II] $k^{2} / 2>1$ and $g_{t t}$ vanishes at the singularity; for others, $g_{t t}$ is infinite at $r=0$.

The nature of region II, which represents the transition from the Schwarzschild-like region I to the singularity of region III, depends strongly on the values of $\Psi_{0}$ and $\omega$. In Schwarzschild, $\alpha=\ln (1-2 / r)$ tends to $-\infty$ as $r \rightarrow 2$, and this is the behavior of $\alpha$ in the nearly Schwarzschild region I; meanwhile, in region II, as in region III, $\alpha$ is well approximated by

$$
\alpha=-\ln r+\alpha_{0} .
$$

The outer boundary of region II is located at the transition between these two behaviors of $\alpha$, i.e., at the minimum of $\alpha$.

Substituting the approximation (33a) for $\alpha$ into the wave equation (31b), we obtain:

$$
\frac{d^{2} \Psi}{d r^{* 2}}=\Psi\left(-\omega^{2}-\frac{e^{2 \alpha_{0}}}{r^{4}}\right) .
$$

Thus, the condition for the scalar field to exhibit spatial oscillations at an approximately constant amplitude is $e^{2 \alpha_{0}} / r^{4} \ll \omega^{2}$. The location where this condition begins to be violated forms the inner boundary of region II. Thus, region II can be said to be defined by the variation of $\alpha$ according to Eq. (33a) as in region III, and by rapid spatial oscillations of the scalar field $d \Psi / d r^{*}=\omega$ as in region I.

Since $\alpha_{0}$ will be more negative for smaller amplitudes of the scalar field, we see that region II is going to be significant for small $\Psi_{0}$, including those in the relevant range of the BBH problem. For larger values of $\Psi_{0}$, the metric and scalar field will proceed directly from region I to region III. 
When region II does exist, the amplitude and phase of the scalar field [solution of Eq. (33b)]

$$
\Psi(r)=A(r) \cos \phi(r)
$$

will be given by

$$
\begin{aligned}
& A=A_{0}\left(1-\frac{e^{2 \alpha_{0}}}{4 r^{4} \omega^{2}}+\ldots\right), \\
& \dot{\phi}=\omega\left(1+\frac{e^{2 \alpha_{0}}}{2 r^{4} \omega^{2}}+\ldots\right),
\end{aligned}
$$

to first order in $e^{2 \alpha_{0}} /\left(r^{4} \omega^{2}\right)$.

Substituting expressions (33) for $\alpha$ and $\Psi$ into the differential equation for $\beta$, Eq. (31d), we find that the dominant term is the first one, $d \beta / d r^{*} \rightarrow$ $(1 / 2) e^{-\alpha_{0}} A^{2} \omega^{2}$, so in region II $\beta$ is approximately

$$
\beta=\frac{1}{2} e^{-\alpha_{0}} A^{2} \omega^{2} r^{*}+\text { const }=\frac{1}{4} e^{-2 \alpha_{0}} r^{2} A^{2} \omega^{2}+\text { const, }
$$

where the last equality comes from the integral of Eq. (31a), $r^{*}=e^{-\alpha_{0}} r^{2} / 2+$ const.

Embedding diagrams and redshifts may provide the best pictorial insight into our full time-averaged standing-wave scalar-field solution, including all of regions I, II and III.

Figure 3 shows an embedding diagram for the standingwave spacetime:

$$
\frac{d z}{d r}=\sqrt{\left|g_{r r}-1\right|}
$$

The 2-surface obtained by rotating around the vertical axis $r=0$ has the same 2-geometry as the surface $(t=$

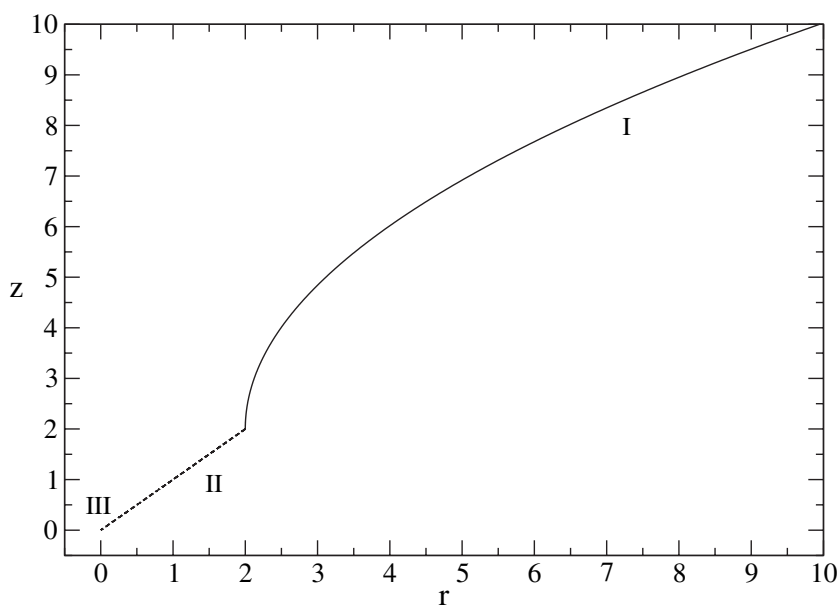

FIG. 3. Embedding diagram for the spacetime with timeaveraged standing-wave scalar field of angular frequency $\omega=$ 0.19 and amplitude $\Psi_{0}=0.015$ at large radii [corresponding to the binary-black-hole separation $a \approx 6 M$; Eq. (12a)]. The solid line represents embedding in Euclidean space; the dashed line, embedding in Minkowski space. Regions I, II and III are labeled on plot. const, $\theta=\pi / 2)$ in the standing-wave spacetime. At radii $r>2$ the embedding is very nearly the same as for a Schwarzschild black hole (cf. Fig. 31.5 of [7]). For $r<$ 2 , the radial distance changes far more slowly than the areal radius $\left(0<g_{r r} \ll 1\right)$, so the embedding is performed in Minkowski space rather than Euclidean space: the metric is $d s^{2}=-d z^{2}+d r^{2}+r^{2} d \phi^{2}$ rather than $d s^{2}=$ $+d z^{2}+d r^{2}+r^{2} d \phi^{2}$. The embedded surface asymptotes to the light cone as $r \rightarrow 0$.

Figure 4 depicts the redshift of light emitted at one radius and received at another, greater one, as a function of the emitting radius:

$$
z=\sqrt{\frac{g_{t t}^{\mathrm{rec}}}{g_{t t}^{\mathrm{em}}}}-1
$$

Figure 4(a) shows that, while the redshift becomes very
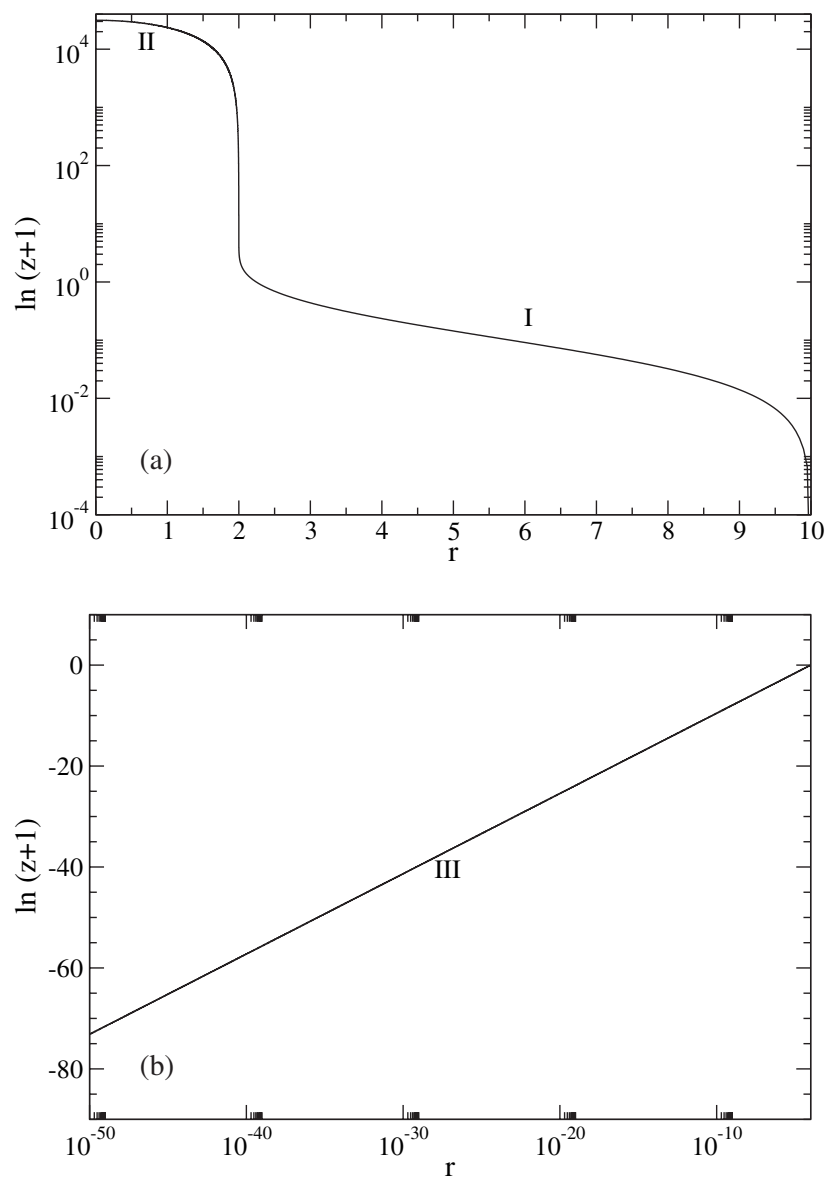

FIG. 4. (a) Redshift $z=\delta \lambda / \lambda$ of light emitted from radius $r$ and received by an observer at $r=10$. (b) Redshift for an observer at $r=0.0001$. A distant observer would see light emitted from $r=0.0001$ redshifted by $\ln (z+1) \approx 10^{5}$. These curves are drawn for the spacetime with time-averaged standingwave scalar field that has angular frequency $\omega=0.19$ and amplitude $\Psi_{0}=0.015$ at large radii [corresponding to the binary-black-hole separation $a \approx 6 M$; Eq. (12a)]. 
large as $r \rightarrow 2$, it never becomes infinite there as it would for a Schwarzschild black hole. As expected, the horizon is destroyed by the standing-wave scalar field, so an observer at infinity can receive signals from any source at $r>0$, albeit with a very large redshift for sources close to or inside the location $(r=2)$ of the Schwarzschild horizon. A blown-up view of the region $r \ll 1$ [Fig. 4(b)] shows that the signal emitted near the singularity may be infinitely redshifted or blueshifted depending on the asymptotics of the scalar field as $r \rightarrow 0$ according to

$$
z=\sqrt{g_{t t}^{\text {rec }}} e^{\left(\alpha_{0}-\beta_{0}\right) / 2} r^{-k^{2} / 4+1 / 2}-1
$$

\section{Comparison of standing-wave and Schwarzschild spacetimes}

It is important to understand how the complete standingwave spacetime with backreaction (we shall call this spacetime $S$ ) compares with the Schwarzschild spacetime (which we shall call spacetime $D$ ). We might first try to compare the metric components in the two spacetimes as functions of the radial coordinate $r$. Indeed, the metric components $g_{\theta \theta}=r^{2}$ and $g_{\phi \phi}=r^{2} \sin ^{2} \theta$ are precisely equal in the two spacetimes when evaluated at the same location in $(t, r, \theta, \phi)$ coordinates. Furthermore, outside the effective-potential region, the perturbation due to the scalar field is so small that the fractional difference $\delta g_{\alpha \beta} / g_{\alpha \beta} \equiv\left(g_{\alpha \beta}^{S}-g_{\alpha \beta}^{D}\right) / g_{\alpha \beta}^{D}$ in metric components $g_{t t}$ and $g_{r r}$ does not exceed $0.01 \%$ for scalar-field parameters in the range of interest. However, the metric components $g_{t t}$ and $g_{r r}$ in $S$ and $D$ can differ by orders of magnitude near $r=2$, inside the effective-potential peak.

The apparent mismatch between the metric components of the two spacetimes near $r=2$ turns out to be a consequence of a poor choice of the radial coordinate $r$ for comparison. A much better choice is $r^{*}$ : when the coordinates $\left(t, r^{*}, \theta, \phi\right)$ are used for mapping between the two spacetimes $S$ and $D$, the metric components agree extremely well near $r=2$.

The fractional differences $\delta g / g$ between the $g_{t t}$ and $g_{\theta \theta}$ components in $S$ and $D$ are plotted in Fig. 5 for scalar-field parameters corresponding to binary-black-hole separations at the boundaries of the range of interest. Using $r^{*}$ rather than $r$ as the coordinate for comparison means that the $g_{\theta \theta}$ components no longer match perfectly; however, the fractional difference introduced remains small as $r \rightarrow 2$ and does not exceed $0.6 \%$ in the range of interest. The fractional differences in $g_{\phi \phi}$ are identical to those in $g_{\theta \theta}$ and are not plotted separately. The Regge-Wheeler tortoise coordinate $r^{*}$ [Eq. (16)] and its generalization [Eq. (31a)] were defined so that $g_{r^{*} r^{*}} \equiv-g_{t t}$ in both spacetimes $S$ and $D$; therefore, the fractional differences in the values of $g_{r^{*} r^{*}}$ in $S$ and $D$ are the same as the fractional differences in $g_{t t}$.

As Fig. 5 shows, the fractional differences in the metrics are $\leqslant 0.02$ down to values of $r^{*} \sim-1000$, a location so deep inside the peak of the effective potential that it con-
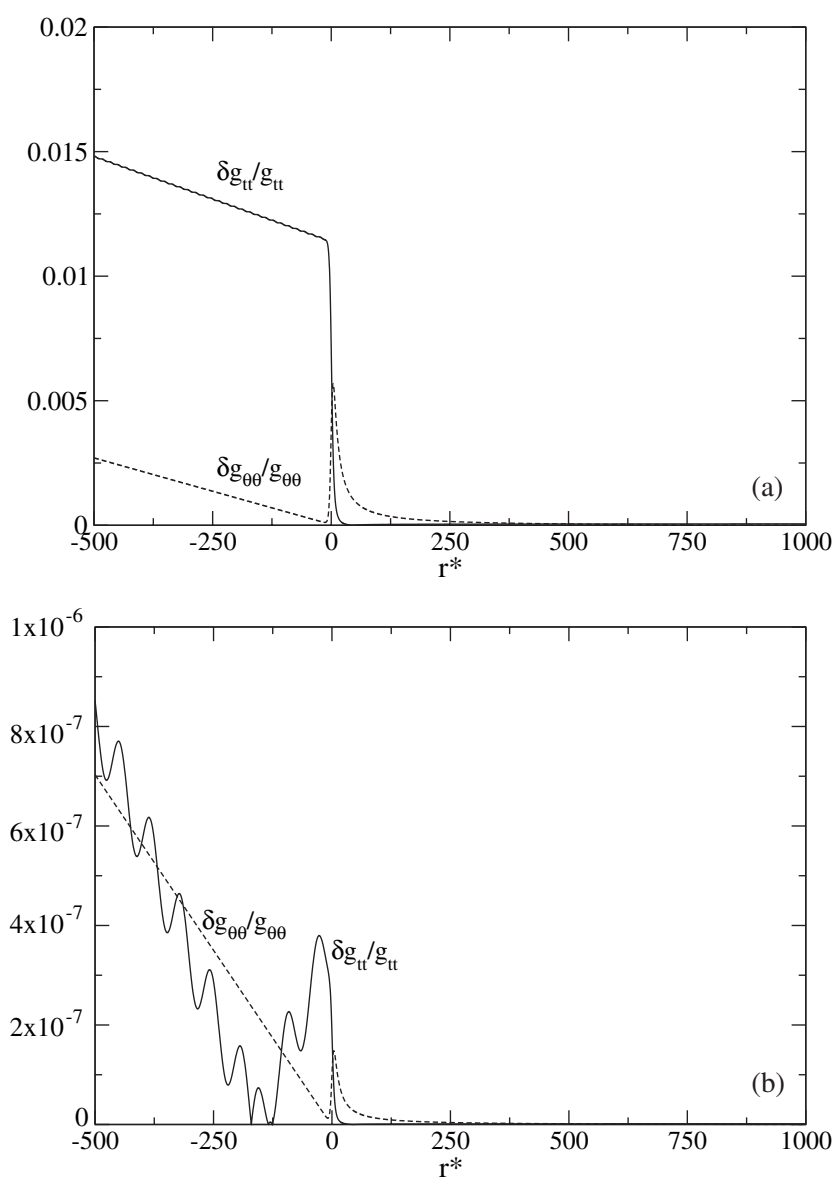

FIG. 5. (a) Fractional differences of the metric components $g_{t t}=-g_{r^{*} r^{*}}$ (solid curve) and $g_{\theta \theta}$ (dashed curve) between Schwarzschild spacetime $D$ and standing-wave scalar-field spacetime $S$ with scalar-wave amplitude and frequency chosen to model BBH separation $a \approx 6 M$ [Eq. (12a)]. (b) Same quantities plotted for scalar-field parameters chosen to model $\mathrm{BBH}$ separation $a \approx 15 M$ [Eq. (12b)].

tains at least 20 near-horizon oscillations of the scalar field for frequencies and amplitudes in the $\mathrm{BBH}$ separation range of interest. Perhaps a more impressive way to state this is that in the $\left(t, r^{*}, \theta, \phi\right)$ coordinate system, metric components of $g^{S}$ and $g^{D}$ match to an accuracy of $2 \%$ for all relevant scalar-field parameters down to the Schwarzschild radius $r^{D}-2<10^{-100}$.

The fractional differences between the coefficients of the metrics $g^{S}$ and $g^{D}$ continue to grow approximately linearly in $r^{*}$ deep inside the effective potential and reach $10 \%$ at the Schwarzschild radius $r^{D}-2 \sim 10^{-3000}$, or approximately 500 scalar-field oscillations inside the effective-potential peak for scalar-field parameters corresponding to BBH separation $a \approx 6 M$.

\section{DOWNGOING SCALAR FIELD}

Having discussed, in Sec. III, the standing-wave scalarfield spacetime that modeled the stationary BBH approxi- 
mation, we now turn to a scalar-field spacetime that serves as a model for the physical BBH spacetime with downgoing gravitational waves at the black-hole horizons: a nearly Schwarzschild spacetime with spherically symmetric scalar waves that are purely downgoing at $r=2$.

For a perturbative analysis of downgoing scalar waves in Schwarzschild, the ingoing, null Eddington-Finkelstein time coordinate $v=t+r^{*}$ is more appropriate than the standard Schwarzschild time coordinate. Let us suppose that by the time $v=0$ a total mass energy $M_{0}=m(v=0)$ is located within the horizon $r=2$. We are not particularly interested in how this mass accumulated there or how the scalar field behaved in the past; we are only interested in the times immediately following $v=0$, and we let the scalar waves be purely downgoing and monochromatic at the horizon for $v>0$. Then for $v>0$ radiation is falling into the black hole at a nearly constant rate, corresponding to the energy density in the scalar field $d m / d v \approx \Psi_{0}^{2} \omega^{2} / 2$, with some small oscillations on top of the linear increase in mass. This is very similar to the Vaidya solution and, indeed, the Vaidya metric will be seen to describe the spacetime of the downgoing scalar-field solution:

$$
d s^{2}=-\left[1-\frac{2 m(v)}{r}\right] d v^{2}+2 d v d r+r^{2} d \Omega^{2} .
$$

Near the horizon, $\Phi=(1 / r) \cos \omega v$ is a purely downgoing solution to the wave equation (1). The only nonzero term of the Ricci tensor in Vaidya coordinates is $R_{v v}=$ $\left(2 / r^{2}\right) m^{\prime}(v)$, where ' denotes the derivative with respect to $v$. The Einstein equations (6) at $r=2$ say:

$$
R_{v v}=\frac{2 m^{\prime}(v)}{4}=2 \Phi_{, v} \Phi_{, v}=\frac{2 \Psi_{0}^{2} \omega^{2} \sin ^{2} \omega v}{4} .
$$

Equation (38) is trivially integrated to obtain:

$$
m(v)=M_{0}+\frac{\Psi_{0}^{2} \omega^{2}}{2} v-\frac{\Psi_{0}^{2} \omega \sin 2 \omega v}{4} .
$$

The black-hole mass grows linearly in $v$ at the rate $\Psi_{0}^{2} \omega^{2} / 2$ with a tiny superimposed oscillatory component. The black hole retains a smooth, nonsingular future horizon.

The scalar field is purely downgoing at the horizon and approximately downgoing everywhere inside the Schwarzschild effective-potential peak. Outside the effective-potential peak there is both a downgoing scalar field and an upgoing one, reflected off the potential. Since for small $v$ the metric is nearly Schwarzschild [the constant Schwarzschild mass $M$ is replaced by the $m(v)$ of Eq. (39)], the scalar field everywhere is given to a high accuracy by a solution to the wave equation in the Schwarzschild background subject to the purely downgoing boundary condition at the horizon. (Of course, very far from the horizon the energy contained in the intervening scalar field will act as an additional mass, but we are not interested in this region for our model problem.)

\section{RECONSTRUCTION OF DOWNGOING SCALAR FIELD FROM STANDING-WAVE SCALAR FIELD}

We turn now to our scalar-wave version of adding a radiation-reaction field to a standing-wave spacetime to obtain a physical spacetime with downgoing waves at horizons and outgoing waves at infinity. For this procedure there is a substantial difference between the $\mathrm{BBH}$ problem and our model problem.

In the true BBH problem, the periodic standing-wave (SW) solution is sourced by the black holes and corresponds to the $\frac{1}{2}$ retarded $+\frac{1}{2}$ advanced solution of the Green's function problem. In this case we add the nonsourced $\frac{1}{2}$ retarded $-\frac{1}{2}$ advanced radiation-reaction (RR) solution of the linearized Einstein equations in the SW spacetime to get an approximation to the physical retarded solution [5]. At infinity, where the SW field is $\frac{1}{2}$ outgoing + $\frac{1}{2}$ ingoing, the boundary condition for the RR field should be set to $\frac{1}{2}$ outgoing $-\frac{1}{2}$ ingoing, so that their sum contains only physical outgoing waves, and similarly at the horizons the RR field will be $\frac{1}{2}$ downgoing $-\frac{1}{2}$ upgoing. Adding this RR field to the $\frac{1}{2}$ downgoing $+\frac{1}{2}$ upgoing standing waves would yield gravitational waves that are downgoing at the expected horizon locations, conforming to the expected behavior in physical black-hole spacetimes. (We do not expect the stress-energy tensor of the sum of SW and RR waves to precisely match the Einstein tensor of the SW spacetime because, of course, gravitational theory is not linear; however, it is likely that "effective linearity" holds in the sense defined by Price [4] for the nontidally locked case as well as for the tidally locked case. In a future paper we intend to explore this issue with a model that more closely resembles the BBH problem.)

The scalar-field model we are currently analyzing is not sourced: the wave equation (1) we used to compute the SW solution is homogeneous. There is then no perturbative homogeneous solution that is simultaneously $\frac{1}{2}$ outgoing $\frac{1}{2}$ ingoing at infinity and $\frac{1}{2}$ downgoing $-\frac{1}{2}$ upgoing at the expected horizon location. Since at the outer boundary the problem is obviously linear for sufficiently weak scalar fields, it is easy to reconstruct the outgoing solution from the SW solution there: we simply double the outgoing component of the SW solution. The interesting case lies in the extraction of a downgoing solution near $r \approx 2$. We attempt to reconstruct the downgoing scalar field from the SW scalar field near the expected horizon by adding to the SW field a perturbative RR field that is $\frac{1}{2}$ downgoing $\frac{1}{2}$ upgoing at $r \approx 2$. We then compare the sum of SW and RR fields to the purely downgoing scalar field obtained in Sec. IV.

As in Sec. III B, let $S$ denote the spacetime of the complete standing-wave solution with backreaction. As discussed in the previous section, the spacetime of the downgoing scalar field is approximated to sufficient accuracy for our purposes by the Schwarzschild spacetime $D$. 
The complete SW scalar field is a solution to the wave equation in spacetime $S$ (in our simplified treatment of the problem, spacetime $S$ actually corresponds to the timeaveraged solution, i.e., one in which we ignore the oscillatory components of the metric). The RR field is a solution to the same wave equation in $S$ in our model. The "reconstructed" downgoing field is, therefore, the downgoing solution to the wave equation in $S$. We want to compare this to the "true" downgoing field, which is the downgoing solution to the wave equation in $D$, i.e., in Schwarzschild.

In the region between the expected horizon location $r=$ 2 and the inner edge of the peak of the effective potential, the wave equation (1) is dominated by

$$
\frac{d^{2} \Psi}{d r^{* 2}} \approx-\omega^{2} \Psi
$$

in both spacetimes $S$ and $D$. Hence, the solution to the wave equation will be oscillatory in $r^{*}$ with frequency $\omega$, which makes sense on physical grounds, since ingoing light cones are $t+r^{*}=$ constant in both $S$ and $D$. Moreover, as discussed in Sec. III B, the metrics of the two spacetimes are nearly the same in the $r^{*}$ coordinate, i.e., $g^{S}\left(r^{*}\right) \approx g^{D}\left(r^{*}\right)$. This suggests that to get the scalarwave phasing to agree, we need to map between the two spacetimes using the $r^{*}$ radial coordinate.

We set the boundary conditions for both the RR field in $S$ and the downgoing field in $D$ at a negative value of $r^{*}$ chosen so that the fields are at least a few wavelengths inside the effective potential, and so that $r^{\mathrm{S}}\left(r^{*}\right)$ is very close to $r^{\mathrm{S}}=2$ (it might actually be slightly inside $r=2$ ). The SW + RR and downgoing scalar fields will match by construction at the point where the initial conditions are set. We will integrate both solutions toward larger $r^{*}$ and compare the quality of the match between the two fields.

For the purposes of comparing the scalar fields in the two spacetimes, we separate the complex scalar field $\Psi\left(r^{*}\right)$ [the spatial factor of the complete field $\Phi(r, t)=$ $\mathfrak{R}\left[\Psi\left(r^{*}\right) e^{-i \omega t}\right] / r$, cf. Eq. (3)] into upgoing and downgoing components. We define the amplitudes and phases of the upgoing and downgoing fields as follows (see below for motivation):

$$
\begin{aligned}
u & \equiv \frac{1}{2 \omega}\left|\frac{d \Psi}{d r^{*}}+i \omega \Psi\right| ; \\
d & \equiv \frac{1}{2 \omega}\left|\frac{d \Psi}{d r^{*}}-i \omega \Psi\right| ; \\
e^{i \phi_{u}} & \equiv \frac{1}{2 i \omega u}\left(\frac{d \Psi}{d r^{*}}+i \omega \Psi\right) ; \\
e^{i \phi_{d}} & \equiv \frac{1}{-2 i \omega d}\left(\frac{d \Psi}{d r^{*}}-i \omega \Psi\right) .
\end{aligned}
$$

To motivate these definitions we consider the geometric optics limit, where the wave phase evolves much faster than the amplitude. In this limit, the downgoing component of the scalar field $\Psi_{d} \propto e^{-i \omega r^{*}}$ separates unambiguously from the upgoing component $\Psi_{u} \propto e^{i \omega r^{*}}$ :

$$
\Psi\left(r^{*}\right)=u e^{i \phi_{u}}+d e^{i \phi_{d}},
$$

where we use the standard approximations

$$
\begin{gathered}
\frac{d \phi_{u}}{d r^{*}} \cong \omega \gg\left|\frac{d u}{d r^{*}}\right|, \\
-\frac{d \phi_{d}}{d r^{*}} \cong \omega \gg\left|\frac{d d}{d r^{*}}\right| .
\end{gathered}
$$

Inverting Eq. (42a) with these approximations yields the definitions (41). Although the geometric optics approximations break down in the region of the effective potential, and the separation of the scalar waves into upgoing and downgoing components becomes ambiguous there because
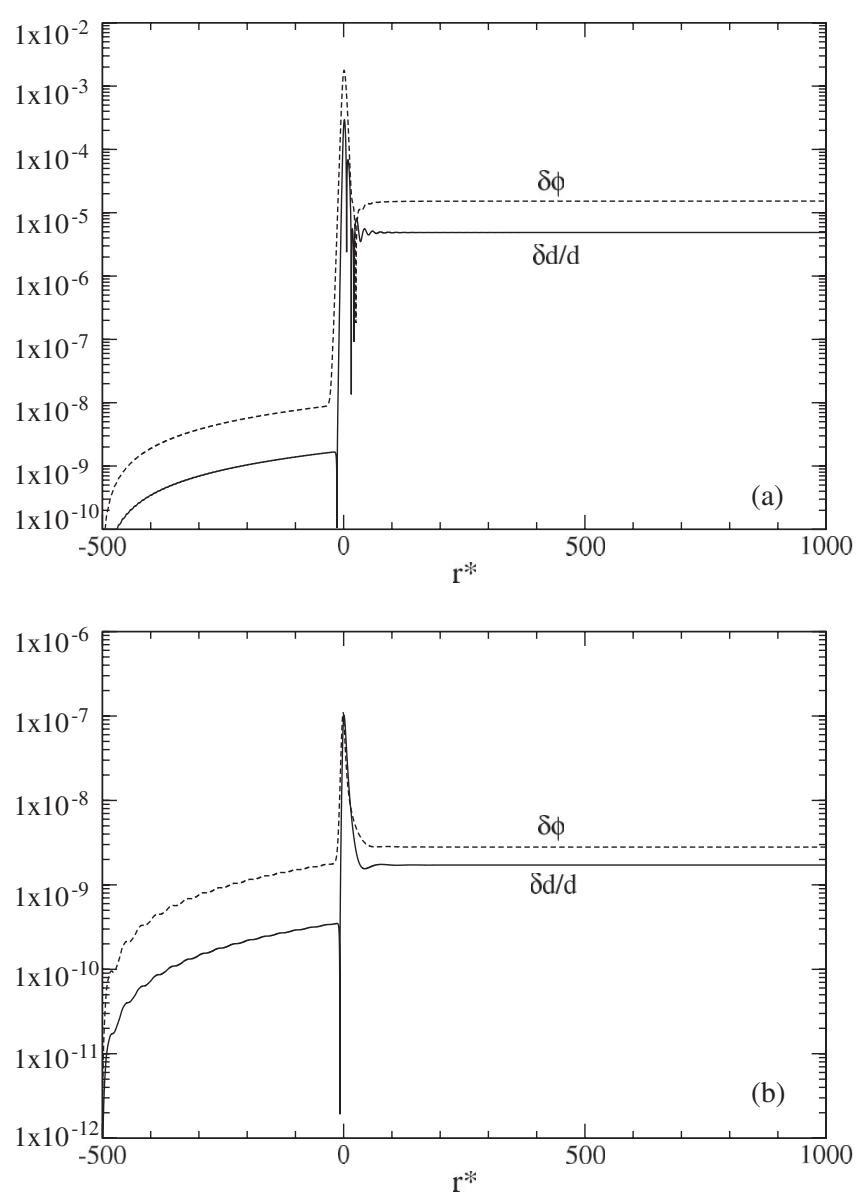

FIG. 6. (a) The fractional difference in the amplitudes of the reconstructed scalar field and downgoing scalar field $\delta d / d \equiv$ $\left(d^{\mathrm{SW}+\mathrm{RR}}-d^{\text {down }}\right) / d^{\text {down }}$ (solid curve) and the phase difference between the two fields $\delta \phi_{d}=\phi_{d}^{\mathrm{SW}+\mathrm{RR}}-\phi_{d}^{\text {down }}$ (dashed curve), plotted vs $r^{*}$. Scalar-wave amplitude and frequency chosen to model BBH separation $a \approx 6 M$. (b) Same quantities plotted for scalar-field parameters chosen to model $\mathrm{BBH}$ separation $a \approx$ $15 M$. 
the wave speed is ill-determined outside the shortwavelength limit, expressions (41) are adequate for comparing scalar fields in our region of interest.

In Fig. 6 we show the fractional difference $\delta d / d \equiv$ $\left(d^{\mathrm{SW}+\mathrm{RR}}-d^{\text {down }}\right) / d^{\text {down }}$ in the amplitude of the downgoing components of the reconstructed $\mathrm{SW}+\mathrm{RR}$ waves and the downgoing waves along with the phase difference $\delta \phi_{d}=\phi_{d}^{\mathrm{SW}+\mathrm{RR}}-\phi_{d}^{\text {down }}$. The two plots represent the end points of the range of relevant BBH separations: $a \approx 6 M$ in Fig. 6(a) and $a \approx 15 M$ in Fig. 6(b). Only the downgoing amplitude $d$ and downgoing phase $\phi_{d}$ are plotted. The upgoing field components are zero to numerical precision inside the effective potential and the differences between the reflected upgoing components of the reconstructed and true downgoing fields outside the effective-potential peak are similar to the differences between the downgoing field components there, $\delta u / u \sim \delta d / d$ and $\delta \phi_{u} \sim \delta \phi_{d}$.

The amplitudes and phases of the true downgoing field and the reconstructed downgoing field match to within one part in ten million from the location where the initial conditions are set (several scalar-field oscillations inside the effective potential) to the inner edge of the effectivepotential peak for all BBH separations in the range of interest. Near the effective-potential peak the fractional difference in the amplitudes does not exceed $0.03 \%$ and the phase difference is less than 0.002 . Outside the effec- tive potential, the fractional difference in the amplitudes is 5 parts per million and the phase difference is less than 0.00002 for the smallest BBH separations in the range of interest.

We also compared the reconstructed and true downgoing fields very deep inside the effective potential when the field-matching initial conditions are set about 10 scalarfield oscillations inside the effective-potential peak. In this case, the amplitudes of the two fields are equal to within numerical precision and the phase difference does not exceed $3 \times 10^{-7}$ down to 500 scalar-field oscillations inside the effective-potential peak. The fields begin to disagree significantly only once the naked singularity is approached in the spacetime $S$, at $r^{S}\left(r^{*}\right) \lesssim 0.2$ [12].

\section{ACKNOWLEDGMENTS}

I am very grateful to my advisor, Kip S. Thorne, for posing this research project and for his extremely patient guidance. Jonathan Gair, Lee Lindblom, Robert Owen, Frans Pretorius and Mark A. Scheel of the TAPIR group at Caltech made many helpful suggestions, as did Ronald J. Adler and Alexander S. Silbergleit of the GP-B theory seminar at Stanford, and Richard Price of UT Brownsville. I was partially supported by NSF Grant No. PHY-0099568 and NASA Grant No. NAG5-12834.
[1] K.S. Thorne, in 300 Years of Gravitation, edited by S. Hawking and W. Israel (Cambridge University Press, Cambridge, England, 1987).

[2] P. R. Brady, J.D. E. Creighton, and K.S. Thorne, Phys. Rev. D 58, 061501 (1998).

[3] S. Detweiler, Phys. Rev. D 50, 4929 (1994).

[4] R. Price, Classical Quantum Gravity 21, S281 (2004); Z. Andrade et al., Phys. Rev. D 70, 064001 (2004).

[5] K. S. Thorne (unpublished).

[6] H. Friedrich, I. Rácz, and R. M. Wald, Commun. Math. Phys. 204, 691 (1999).

[7] C. W. Misner, K. S. Thorne, and J. A. Wheeler, Gravitation (Freeman, San Francisco, 1973).

[8] V.P. Frolov and I.D. Novikov, Black Hole Physics, Fundamental Theories of Physics (Kluwer, Dordrecht,
1998).

[9] E. Poisson and M. Sasaki, Phys. Rev. D 51, 5753 (1995).

[10] H. Fang and G. Lovelace, gr-qc/0505156.

[11] T. Regge and J. A. Wheeler, Phys. Rev. 108, 1063 (1957).

[12] It might seem odd that the fields continue to match far deeper (at far more negative $r^{*}$ ) than the metrics, which begin to disagree by $10 \%$ at 500 scalar-field oscillations inside the effective-potential peak. The reason is that in the wave equation, the metric enters only into the effective-potential piece [see Eq. (31b)], which is so tiny throughout the region $0.2 \lesssim r^{S} \lesssim 2$ that even significant deviations of the standing-wave spacetime metric $g^{S}$ from the Schwarzschild metric do not affect the behavior of the scalar field. 